\title{
Faith Healer and Cultural Belief to Development of Psychopathology: A Case Report
}

\author{
Iftikar Hussain ${ }^{1}$, Soumik Sengupta ${ }^{2}$, Sucheta Saha ${ }^{3}$
}

\begin{abstract}
The presentation of mental illness and understanding of the patient's experience with regard to psychopathology is quite unique and cannot be generalized due to the diverse etiological factors. In understanding and managing psychopathology, cultural knowledge plays a vital role. It has been seen that most of the interventions, in a similar fashion, need an understanding of historical roots in the specific cultural background and they also shape the psychotherapy models. The current case shows us the development of psychopathology with regard to cultural belief as well as discerning the symptom profile associated with it. $\mathrm{Mr} \mathrm{X}$, a 41-year-old gentleman from a rural area of Assam, presented with complaints about a"living thing" inhabiting his abdomen for the last 2 years and has been growing ever since. He claims that an enraged faith healer with incredible powers had done so to him. He was diagnosed with persistent delusional disorder at LGBRIMH and was managed with both pharmacological and nonpharmacological interventions. In nonpharmacological interventions, coping strategies and insight-oriented psychotherapy in context to his cultural beliefs were given. Significant improvement was seen after 2 months and at 3 months his socio-occupational functioning improved as well. Keywords: Cultural beliefs, Faith healer, Psychopathology.

Indian Journal of Private Psychiatry (2019): 10.5005/jp-journals-10067-0026
\end{abstract}

\section{INTRODUCTION}

Understanding the dynamic interplay of cultural forces can be an eye-opener to how people manifest symptoms, cope with psychological challenges, and how it aids in accurate diagnosis. Witchcraft and black magic, Godmen, faith healing, and animal sacrifice, etc., have been part and parcel of Indian superstitious beliefs and have dominated the content of delusions and hallucinations. Although in the last few decades, researchers have tried to examine cultural influences in psychopathology, it still remains as a marginal field of inquiry.

\section{Case Description}

Mr X, a 41-year-old married Hindu farmer hailing from a rural area of Assam belonging to a lower socio-economic background who completed schooling up to grade four, presented with complaints about a living "thing" which has been residing in his abdomen for the last 2 years.

As corroborated by the family members, the patient recalls that 2 years back a stranger had visited his village who claimed to have powers to predict future and avert dangers, if any. It is a popular belief in their local culture that faith healers can foresee future dangers and provide miraculous solutions. He went to the mysterious healer who then showed him four white cards and asked him to touch them. The cards turned red on his touch (verbatim of the patient). On seeing this, the stranger said that he is going to suffer from some kind of calamity in near future, but $\mathrm{Mr} \mathrm{X}$ need not worry as he could prevent it by conducting a set of rituals. But due to financial constraints our patient was not able to do so and the stranger left without performing the rituals. After a few weeks, he started saying that the stranger had turned wrathful and he had put something inside his abdomen by unknown means. He firmly believes that it is a "living thing" which is growing toward his head and this will be the cause of his untimely demise. After "it" has consumed him, it will move onto inhabit his wife and she

\footnotetext{
${ }^{1-3}$ Department of Psychiatry, Lokopriya Gopinath Bordoloi Regional Institute of Mental Health, Tezpur, Assam, India

Corresponding Author: Iftikar Hussain, Department of Psychiatry, Lokopriya Gopinath Bordoloi Regional Institute of Mental Health, Tezpur, Assam, India, Phone: +91 8638951606, e-mail: ifti.amc@gmail. com

How to cite this article: Hussain I, Sengupta S, Saha S. Faith Healer and Cultural Belief to Development of Psychopathology: A Case Report. Ind J Priv Psychiatry 2019;13(1):22-23.

Source of support: Department of Psychiatry, Lokopriya Gopinath Bordoloi Regional Institute of Mental Health, Tezpur, Assam, India Conflict of interest: None
}

too will meet with similar fate. Since then he has visited multiple faith healers to take out that "thing" but, as per him, none of them could. He started having low mood, poor sleep, and appetite for the last 6 months or so. Now patient seems self-absorbed most of the times and does not have any zeal for work or life. Exhausting all possible solutions, his family then decided to seek medical help and hence the visit to LGBRIMH OPD.

Physical examination as well as relevant blood and radiological examinations did not reveal any abnormalities. Mental status examination revealed anxious effect, somatic delusion in the form of "living thing" inside his abdomen, which he strongly believes and not prevalent in their culture and cannot be reasoned out. Diagnosis was according to ICD-10 kept as persistent delusional disorder (F22.0). Assessments-brown assessment of beliefs scale (BABS) - total score was 21.

Management-patient was started on risperidone $4 \mathrm{mg}$ per day with nonpharmacological management in the form of insightoriented psychotherapy as well as coping strategies. On subsequent follow-up for 1 month, the dosage of risperidone increased to $6 \mathrm{mg}$ per day after assessing the symptoms. He is on regular follow-up and on regular assessment. His biological functions and functionality improved after around 5 weeks of treatment and his distress for the 
delusion is also improved after 2 months of treatment. At 3 months, he showed significant improvement in social and occupational functioning and his BABS score was reduced to 6 .

\section{Discussion}

Currently a little is known about the interaction between culture and the formation of delusions. ${ }^{1}$ Culture that includes processes that operate at the individual and social levels can play an important role in adaptation and maladaptation. ${ }^{2}$ Sociocultural factors such as religious belief systems $\mathrm{s}^{3,7,8}$ and more collective or individualistic conceptualizations of self ${ }^{10}$ as well as environmental factors, ${ }^{10}$ socio-economic status, ${ }^{4,5}$ geography, ${ }^{6,9}$ and urban and rural living contexts ${ }^{3,4}$ have been shown to influence the content of delusions. In our case, we found the development of delusion closely related to the cultural belief specifically on faith healer.

\section{CONCLUSION}

Certain mental-health issues cross cultural boundaries while others seem to confine within specific groups of people. Whatever be the distribution, the stability and universality within categories are astounding. So if such factors help in tracing down the development of psychopathology, it helps in individualizing the treatment and also ascertains a better response to the same. Continued research into the content of delusions within the specific geographical areas will be helpful in the field of psychiatric practise.

\section{Ethics Approval and Consent to Participate}

Personal details are kept confidential. Approval from the ethical committee as well as from the patient was taken.

\section{References}

1. Gold J, Gold I. The "Truman Show" delusion: psychosis in the global village. Cogn Neuropsychiatry 2012;17(6):455-472. DOI: 10.1080/ 13546805.2012.666113.

2. Causadias JM. A roadmap for the integration of culture into developmental psychopathology.Developmentand Psychopathology 2013;25:1375-1398. DOI: 10.1017/S0954579413000679.

3. Stompe T, Friedman A, Ortwein G, et al. Comparison of delusions among schizophrenics in Austria and in Pakistan. Psychopathology 1999;32(5):225-234. DOI: 10.1159/000029094.

4. Kala A, Wig N. Delusion across cultures. Int J Soc Psychiatry 1982;28(3):185-193. DOI: 10.1177/002076408202800304.

5. Suhail K. Phenomenology of delusions in Pakistani patients: effect of gender and social class. Psychopathology 2003;36(4):195-199. DOI: 10.1159/000072789.

6. Gecici O, Kuloglu M, Guler O, et al. Phenomenology of delusions and hallucinations in patients with schizophrenia. Klin Psikofarmakol B 2010;20(3):204-212. DOI: 10.1080/10177833.2010. 11790661.

7. Bhavsar V, Bhugra D. Religious delusions: finding meanings in psychosis. Psychopathology 2008;41(3):165-172. DOI: 10.1159/ 000115954.

8. Stompe T, Bauer S, Ortwein-Swoboda G, et al. Delusions of guilt: the attitude of Christian and Muslim schizophrenic patients toward good and evil and the responsibility of men. J Muslim Ment Health 2006;1(1):43-56. DOI: 10.1080/15564900600654294.

9. Tateyama $M$, Asai $M$, Hashimoto $M$, et al. Transcultural study of schizophrenic delusions. Psychopathology 1998;31(2):59-68. DOI: 10.1159/000029025.

10. Suhail K, Cochrane R. Effect of culture and environment on the phenomenology of delusions and hallucinations. Int J Soc Psychiatry 2002;48(2):126-138. DOI: 10.1177/002076402128783181. 